\title{
Termoterapia de Banana 'Prata-Anã' no Controle de Podridões em Pós-Colheita*
}

\author{
Wilson da S. Moraes ${ }^{1 * *}$, Laércio Zambolim² ${ }^{\text {, Juliana D. Lima }}{ }^{3}$, Luiz C. C. Salomão ${ }^{4} \&$ Paulo Cecon $^{5}$ \\ ${ }^{1}$ Polo Regional do Vale do Ribeira/Agência Paulista de Tecnologia dos Agronegócios - APTA, Av. Wild José de Souza, 454, \\ Centro, Registro, SP, CEP 11900-000, Fax: (013) 3821-2282, e-mail: wilson_moraes@uol.com.br; ${ }^{2}$ Departamento de \\ Fitopatologia, Universidade Federal de Viçosa; ${ }^{3}$ Unidade Diferenciada de Registro, Universidade Estadual Paulista; \\ ${ }^{4}$ Departamento de Fitotecnia, Universidade Federal de Viçosa; ${ }^{5}$ Departamento de Estatística, Universidade Federal de Viçosa
}

(Aceito para publicação em 04/05/2005)

Autor para correspondência: Wilson da Silva Moraes

MORAES, W.S., ZAMBOLIM, L., LIMA, J.D., SALOMÃO, L.C.C. \& CECON, P. Termoterapia de banana 'Prata-Anã' no controle de podridões em pós-colheita. Fitopatologia Brasileira 30:603-608. 2005.

\section{RESUMO}

O objetivo desse estudo foi determinar a tolerância de banana (Musa spp.) 'Prata-Anã' (AAB) e do fungo Colletotrichum musae à termoterapia no controle de podridões em pós-colheita. Experimentos in vivo e in vitro foram instalados em delineamento inteiramente casualizado, seguindo um esquema fatorial $4 \times 5$ (temperatura $\mathrm{x}$ tempo). Os tratamentos consistiram na imersão dos frutos (buquês) e do fungo (esporos e micélio) em água aquecida a 47, 50, 53 e $56{ }^{\circ} \mathrm{C}$, durante $0,3,6,9$ e 12 min. A exposição dos frutos a $56{ }^{\circ} \mathrm{C}$ durante 9 min causou escurecimento da casca nas extremidades dos frutos, porém, as características físicas e químicas dos frutos não foram alteradas pelos tratamentos. Frutos inoculados e tratados a $56^{\circ} \mathrm{C}$ durante 6 min não apresentaram podridões nem escurecimento da casca, enquanto aqueles não tratados apresentaram $64 \%$ da área lesionada / fruto. A partir das combinações $53{ }^{\circ} \mathrm{C} / 9 \mathrm{mi}$. e $56{ }^{\circ} \mathrm{C} / 3 \mathrm{~min}$ a germinação de esporos foi reduzida para $4 \%$ e $0 \%$, respectivamente. A combinação $56{ }^{\circ} \mathrm{C} / 12$ min reduziu, mas não paralisou o crescimento micelial. $\mathrm{O}$ tratamento $56{ }^{\circ} \mathrm{C} / 6 \mathrm{~min}$ retardou mas não paralisou o crescimento micelial in vitro, porém foi efetivo no controle completo das podridões in vivo. Esse tratamento evitou a manifestação de podridões no inverno (maio), mas não no verão (novembro), mostrando-se influenciado pelas condições climáticas próximas à colheita dos cachos. A termoterapia pode ser recomendada para controle de podridão em pós-colheita de banana devendo ser ajustada para diferentes estações do ano.

Palavras-chave adicionais: Colletotrichum musae, antracnose, infecção latente.

\begin{abstract}
Thermotherapy of 'Prata anã' Banana in postharvest rot control

The objective of this study was to determine the tolerance of 'Prata-Anã' (AAB) banana (Musa spp.) fruits and of Colletotrichum musae to thermotherapy in postharvest rot control. In vivo and in vitro experiments were installed in a completely randomized design, arranged in a $4 \times 5$ factorial scheme (temperature $\mathrm{x}$ time immersion). Treatments were the immersion of fruits and fungus (spores and mycelium) in water heated at 47, 50, 53 and $56{ }^{\circ} \mathrm{C}$ from $0,3,6,9$ and 12 min. The exposure of fruits at $56^{\circ} \mathrm{C}$ for 9 min caused blackening in fruit extremities. Chemical and physical fruit characteristics were not changed by treatments. Inoculated and treated fruits at $56{ }^{\circ} \mathrm{C}$ during 6 min did not show rot and peel blackening, while non-treated fruits have shown $64 \%$ of lesions/fruit. From the combinations $53{ }^{\circ} \mathrm{C} / 9 \mathrm{~min}$ and $56{ }^{\circ} \mathrm{C} / 3 \mathrm{~min}$, spore germination was reduced to $4 \%$ e $0 \%$ respectively. The combination $56{ }^{\circ} \mathrm{C} / 12$ min retarded but did not stop mycelial growth. Treatments $56{ }^{\circ} \mathrm{C} / 6 \mathrm{~min}$ did not stop mycelial growth in vitro, but was effective in vivo rot control. This treatment avoided rot manifestation in the winter (May) but did not in the summer (November), being influenced by environmental conditions near to bunch harvest. Thermotherapy can be recommended for banana postharvest rot control adapted to different seasons of the year.
\end{abstract}

Additonal keywords: Colletotrichum musae, anthracnose, latent infecction.

\section{INTRODUÇÃO}

A bananicultura (Musa spp.) destaca-se como atividade de grande importância econômica e social, que em 2002 posicionou o Brasil como segundo maior produtor mundial (FAO, 2004). Apesar disso, existem dificuldades

\footnotetext{
*Parte da Tese de Doutorado do primeiro autor apresentada à Universidade Federal de Viçosa. (1999)

***Bolsista da CNPq
}

na comercialização porque o fruto de banana é altamente perecível e predisposto a sérias perdas em pós-colheita, principalmente devido ao estádio impróprio de maturação do fruto, as práticas inadequadas de colheita e armazenamento e às doenças em pós-colheita (Ramma et al., 1999).

As principais doenças que ocorrem em pós-colheita de banana são a antracnose causada por Colletotrichum musae (Berk. \& Curtis) Arx (Gloesporium musarum Cook $\&$ Massee) e a podridão da coroa causada por um complexo 
de fungos (Fusarium pallidoroseum (Cooke) Sacc. $=$ Fusarium semitectum Berk. \& Ravenel, Fusarium moniliform J. Sheld e Colletotrichum musae Berk \& Curtis) (Ploetz el al, 1994). Outros patógenos também podem estar associados aos frutos como Chalara paradoxa $($ De Seyn) Sacc $=$ Thielaviopsis paradoxa (De Seyn) Höhn, Botryodiplodia theobromae Pat. e Verticillium theobromae (Turconi) Mason \& Hughes, causando podridão do engaço, podridão do fruto e ponta de charuto, respectivamente (Reyes et al., 1998).

A principal estratégia de controle das doenças em póscolheita de muitos frutos é o uso de fungicidas. Entretanto, a forma de aplicação, o surgimento de patógenos resistentes e as pressões sócio-econômicas têm reduzido as oportunidades de planejar estratégias de controle baseadas em fungicidas, culminando com a retirada dos registros de muitos produtos do mercado (Johnson \& Sanghote, 1994). Assim, surge a necessidade de desenvolver métodos alternativos de controle que não comprometam a qualidade dos frutos e a saúde humana.

A termoterapia inicialmente utilizada em tratamentos quarentenários (Couey, 1989), é um método alternativo de controle de doenças em pós-colheita de vários frutos (Shiffmann-Nadel \& Cohen, 1966; Jesus et al., 1994; Jacobi \& Giles, 1997), capaz de erradicar ou enfraquecer o patógeno (Golan \& Phillips, 1991), reduzir desordens fisiológicas na armazenagem (Jacobi \& Giles, 1997) e manter os frutos livres de pesticidas (Liu et al., 1997).

A eliminação do patógeno pode ser obtida com temperatura e tempo próximos a produzir efeitos deletérios no fruto (Armstrong, 1982). Por isso, a sensibilidade térmica do fruto e do patógeno deve ser diferente para aumentar o sucesso do tratamento (Barkai-Golan \& Phillips, 1991).

A imersão de frutos em água aquecida de 50 a $55^{\circ} \mathrm{C}$, por $10 \mathrm{~min}$, tem sido considerado método padrão para controle pós-colheita de várias doenças fúngicas (Golan \& Phillips, 1991; Liu et al., 1997). Curtos tempos de exposição em temperaturas mais elevadas mostram-se mais efetivos em alterar a temperatura superficial dos frutos; assim, podem erradicar o patógeno presente no interior da casca dos frutos (Golan \& Phillips, 1991).

A tolerância de frutos à termoterapia foi estabelecida para variedades de bananas do subgrupo Cavendish (AAA) (Burden, 1968; Rahman et al., 1994) e 'Prata de Santa Catarina' (AAB) (Reyes et al., 1998) para controle da antracnose e podridão da coroa, respectivamente, e para o controle da mosca das frutas (Armstrong, 1982; Couey, 1989). Porém, foram observados danos ou injúrias, principalmente, escaldaduras da casca nas extremidades dos frutos (Reyes et al., 1998).

Diante da escassez de informações sobre termoterapia em banana 'Prata anã', este estudo teve por objetivo determinar o efeito da termoterapia in vivo e in vitro no controle de podridões em pós-colheita de banana 'Prata anã'.

\section{MATERIAL E MÉTODOS}

Cachos de banana 'Prata-Anã' foram colhidos em estádio pré-climatérico de plantios de dois anos de idade localizadas em Jaíba, MG. Em seguida, foram despencados, sendo as pencas centrais do cacho selecionadas e acondicionadas em caixas plásticas, protegidas do sol e transportadas 12 h em caminhão aberto, até o Laboratório de Pós-Colheita da Universidade Federal de Viçosa.

$\mathrm{O}$ tratamento dos buquês (cinco frutos) foi realizado em caixa d'água de 2501 , contendo um sistema de aquecimento de água com quatro resistências elétricas de 1500 watts (totalizando 6000 watts), uma bomba d'água de $1 / 2 C V$ e um termostato. A temperatura da água foi monitorada com termômetro digital.

Foram instalados experimentos in vivo e in vitro nos quais os tratamentos consistiram na imersão dos frutos, esporos ou micélio de $C$. musae em água a $47,50,53$ e $56^{\circ} \mathrm{C}$, por zero, três, seis, nove e $12 \mathrm{~min}$, seguida do resfriamento em água a 20 ${ }^{\circ} \mathrm{C}$. Experimentos in vivo foram realizados na caixa térmica acima referida e os experimentos in vitro, em banho-maria.

\section{Experimento I - Efeito da termoterapia sobre o amadurecimento dos frutos}

Após os tratamentos, os buquês foram secos ao ar, colocados em caixas plásticas forradas com papel picado e mantidos em galpão anexo ao laboratório, sob condições ambientes ( 18 a $23{ }^{\circ} \mathrm{C}$ e 60 a $85 \%$ UR). Para cada tratamento foram utilizados quatro caixas contendo quatro buquês, cada uma servindo como repetição. Decorrido 12 dias dos tratamentos, amostras de frutos foram retiradas para análises físicas e visuais (injúrias na casca, temperatura da polpa, evolução do peso, cor e firmeza) e químicas ( $\mathrm{pH}$, acidez e teor de clorofilas e carotenóides).

Adicionalmente, a temperatura central da polpa foi monitorada com termopar digital. Os dados obtidos foram submetidos à análise de regressão, cujo modelo foi definido pela significância dos coeficientes e pelo coeficiente de determinação.

\section{Experimento II - Efeito da termoterapia sobre a germinação de esporos de $C$. musae}

Uma suspensão de esporos foi obtida de colônias de $C$. musae cultivadas em BDA, provenientes de bananas naturalmente infetadas. Três gotas dessa suspensão foram transferidas para os tubos de ensaio, mantidos em banho-maria nas condições anteriormente definidas. Após os tratamentos, uma alíquota de $1 \mathrm{ml}$ da suspensão de esporos foi vertida em placas de Petri contendo ágar-água e incubadas a $25^{\circ} \mathrm{C}$ por 48 h. Para cada tratamento, foram utilizadas três placas de Petri como repetição. As avaliações foram realizadas 48 h após os tratamentos, determinando-se a percentagem de esporos germinados, cujo tubo germinativo atingiu pelo menos o dobro do comprimento do esporo.

\section{Experimento III - Efeito da termoterapia sobre o crescimento micelial de $C$. musae}

Colletotrichum musae isolado de frutos naturalmente infetados foi cultivado em BDA, durante cinco dias. Posteriormente, três discos de micélio foram transferidos para 
tubos de ensaio contendo $1 \mathrm{ml}$ de água esterilizada, mantidos em banho-maria nas condições anteriormente definidas. Em seguida, os discos foram transferidos para placas de Petri contendo BDA e incubados a $25^{\circ} \mathrm{C}$, durante seis dias. Para cada tratamento foram utilizadas três placas como repetição. $\mathrm{O}$ crescimento radial foi determinado pelo diâmetro médio da colônia, avaliado seis dias após os tratamentos.

\section{Experimento IV - Efeito da termoterapia sobre a infecção latente causada por $C$. musae}

Os buquês foram atomizados com uma suspensão de esporos de C. musae, obtida de colônias monospóricas cultivadas em BDA durante sete dias, ou aspergidos com água esterilizada (controle). Posteriormente, foram incubados em câmara úmida a $25{ }^{\circ} \mathrm{C}$ por $24 \mathrm{~h}$ e, imediatamente, submetidos à termoterapia nas condições definidas anteriormente. Após os tratamentos, os buquês foram colocados em caixas plásticas, mantidas em galpão anexo ao laboratório, sob condições ambientes ( 18 a $23{ }^{\circ} \mathrm{C}$ e 60 a $85 \%$ UR). Para cada tratamento foram utilizadas quatro caixas contendo quatro buquês, cada uma servindo como uma repetição. Avaliou-se o tempo necessário para manifestação das podridões e a severidade da doença aos 12 dias após os tratamentos. A severidade foi determinada pela percentagem da área lesionada por fruto (\% ALF), utilizando-se escala diagramática desenvolvida para esse fim, na qual a área lesionada variou de 0 a $64 \%$.

Os experimentos in vivo (I e IV) e in vitro (II e III) foram dispostos em delineamento inteiramente casualizado, seguindo esquema fatorial $4 \times 5$ (quatro temperaturas e cinco tempos) com quatro e três repetições, respectivamente. Os dados foram submetidos à análise de variância pelo teste $\mathrm{F}$ e, quando significativo, as médias dos tratamentos foram comparadas pelo teste de Tukey ao nível de 5\% de probabilidade.

Os experimentos foram implantados e conduzidos em maio (inverno). A partir dos resultados obtidos, implantouse outro experimento em novembro (verão), seguindo a mesma metodologia dos experimentos realizados in vivo, quando se avaliou a manifestação de injúrias e de podridões para ratificar a eficiência da combinação temperatura $\mathrm{x}$ tempo sob condições mais quentes.

\section{RESULTADOS E DISCUSSÃO}

A termoterapia a $56^{\circ} \mathrm{C}$, a partir de 9 min de exposição, provocou injúrias nas extremidades dos frutos caracterizadas pelo escurecimento da casca. Injúrias na casca também foram observadas por Armstrong (1982) em bananas tratadas a 55 ${ }^{\circ} \mathrm{C}$ e por Rahman et al. (1994) em bananas tratadas acima de $50{ }^{\circ} \mathrm{C}$, principalmente nas extremidades dos frutos. Banana 'Prata de Santa Catarina' (Musa AAA) tratada a $55{ }^{\circ} \mathrm{C}$ por 10 min ou mais, apresentou escaldaduras na casca e endurecimento da polpa (Reyes et al., 1998).

Para Shiffmann-Nadel \& Cohen (1966), as injúrias causadas pela termoterapia incluem o aumento na perda de peso, descoloração da casca, aumento da suscetibilidade a fungos e a redução da vida pós-colheita e são caracterizadas pela falta de desenvolvimento normal da pigmentação, amolecimento anormal e declínio na produção de etileno. Assim, a taxa respiratória e a síntese de etileno são afetadas pela exposição a elevadas temperaturas.

No presente estudo, as injúrias na casca foram observadas apenas nas extremidades dos frutos expostos à temperatura de $56^{\circ} \mathrm{C}$ a partir de $9 \mathrm{~min}$, não sendo constatado, portanto, nenhuma descoloração ou retardamento do amadurecimento. No final das avaliações, 12 dias após os tratamentos, não houve efeito significativo dos fatores isolados, temperatura e tempo, nem da interação desses fatores, na perda de peso, coloração externa da casca, firmeza, $\mathrm{pH}$, acidez, teores de clorofilas e carotenóides, segundo teste $\mathrm{F}(\mathrm{P}<0,05)$ da análise de variância (Tabela 1).

Segundo Bleinroth (1992), no momento da colheita, os frutos apresentam, em geral, elevado metabolismo e máxima taxa respiratória; assim, a temperatura interna dos frutos apresenta-se 5 a $9 \%$ acima da temperatura ambiente. Os resultados demonstraram que a temperatura central da polpa dos frutos $(Y)$, imediatamente após a termoterapia, aumentou proporcionalmente com a temperatura da água $(T)$ e tempo de exposição $(M)$, definida pelo modelo $Y=23,6275$ $1,70513 * M+0,0670737 * T M$, atingindo $42^{\circ} \mathrm{C}$ de temperatura central da polpa a $56{ }^{\circ} \mathrm{C}$, durante 9 min de exposição, no qual verificaram-se injúrias na casca dos frutos (Figura 1).

A percentagem de esporos germinados 48 h após a termoterapia foi significativamente influenciada pela interação da temperatura da água e o tempo de exposição. O tratamento $53{ }^{\circ} \mathrm{C}$, a partir de $9 \mathrm{~min}$, e $56{ }^{\circ} \mathrm{C}$, a partir de 3 min, reduziram a percentagem de germinação de esporos para 0\% (Tabela 2). A resposta de um patógeno a termoterapia pode ser influenciada pelo conteúdo de umidade dos esporos, pela atividade metabólica do patógeno e pela idade ou composição química do inóculo. Assim, os esporos germinados mostram-se mais sensíveis do que aqueles não germinados. Algumas diferenças genéticas são expressas por variações na sensibilidade a elevadas temperaturas (Golan \& Phillips, 1991).

$\mathrm{O}$ crescimento micelial do fungo C. musae, seis dias após a termoterapia, mostrou-se significativamente influenciado tanto pela interação temperatura da água e tempo de exposição, como por esses fatores isolados. Apenas o tratamento $56{ }^{\circ} \mathrm{C}$ por 12 min mostrou-se efetivo em retardar o crescimento micelial, mas não paralisar o crescimento do fungo (Tabela 3).

Após a determinação da combinação temperatura da água $\left(56^{\circ} \mathrm{C}\right)$ e tempo de exposição $(9 \mathrm{~min})$ responsável por injúrias na casca dos frutos, partiu-se para a definição da combinação capaz de controlar as infecções latentes ou podridões em frutos de banana inoculados artificialmente. Os resultados mostraram que a percentagem da área lesionada por fruto (\% ALF) 12 dias após a termoterapia foi influenciada significativamente, tanto pela interação da temperatura e tempo de exposição, como por esses fatores isolados. Os 
W.S. Moraes et al.

TABELA 1 - Alterações nas características físicas e químicas de frutos de banana (Musa spp.) 'Prata anã' submetidos à termoterapia e incubados sob condições ambientes, em função da temperatura e do tempo de imersão, 12 dias após o tratamento

\begin{tabular}{lccccccc}
\hline \hline Temperatura $\left({ }^{\circ} \mathbf{C}\right)$ & Perda de Peso & Cor & Firmeza & pH & Ácido Málico & Clorofila & Carotenóide \\
\hline 47 & $15,08^{\mathrm{ns}}$ & $6,40^{\mathrm{ns}}$ & $1,04^{\mathrm{ns}}$ & $4,69^{\mathrm{ns}}$ & $0,65^{\mathrm{ns}}$ & $1,89^{\mathrm{ns}}$ & $0,41^{\mathrm{ns}}$ \\
50 & $14,53^{\mathrm{ns}}$ & $6,25^{\mathrm{ns}}$ & $1,07^{\mathrm{ns}}$ & $4,59^{\mathrm{ns}}$ & $0,72^{\mathrm{ns}}$ & $2,43^{\mathrm{ns}}$ & $0,43^{\mathrm{ns}}$ \\
53 & $14,33^{\mathrm{ns}}$ & $6,48^{\mathrm{ns}}$ & $1,05^{\mathrm{ns}}$ & $4,78^{\mathrm{ns}}$ & $0,69^{\mathrm{ns}}$ & $2,14^{\mathrm{ns}}$ & $0,44^{\mathrm{ns}}$ \\
56 & $14,28^{\mathrm{ns}}$ & $6,56^{\mathrm{ns}}$ & $1,05^{\mathrm{ns}}$ & $4,68^{\mathrm{ns}}$ & $0,70^{\mathrm{ns}}$ & $2,33^{\mathrm{ns}}$ & $0,44^{\mathrm{ns}}$ \\
\hline DMS & 0,90 & 0,35 & 0,05 & 0,29 & 0,09 & 0,92 & 0,08 \\
\hline Tempo (minutos) & & & & & & & \\
\hline 0 & $14,88^{\mathrm{ns}}$ & $6,68^{\mathrm{ns}}$ & $1,06^{\mathrm{ns}}$ & $4,63^{\mathrm{ns}}$ & $0,71^{\mathrm{ns}}$ & $2,15^{\mathrm{ns}}$ & $0,46^{\mathrm{ns}}$ \\
3 & $14,94^{\mathrm{ns}}$ & $6,40^{\mathrm{ns}}$ & $1,05^{\mathrm{ns}}$ & $4,66^{\mathrm{ns}}$ & $0,69^{\mathrm{ns}}$ & $1,99^{\mathrm{ns}}$ & $0,42^{\mathrm{ns}}$ \\
6 & $14,48^{\mathrm{ns}}$ & $6,35^{\mathrm{ns}}$ & $1,06^{\mathrm{ns}}$ & $4,66^{\mathrm{ns}}$ & $0,69^{\mathrm{ns}}$ & $2,46^{\mathrm{ns}}$ & $0,44^{\mathrm{ns}}$ \\
9 & $14,65^{\mathrm{ns}}$ & $6,34^{\mathrm{ns}}$ & $1,07^{\mathrm{ns}}$ & $4,64^{\mathrm{ns}}$ & $0,68^{\mathrm{ns}}$ & $2,48^{\mathrm{ns}}$ & $0,44^{\mathrm{ns}}$ \\
12 & $13,89^{\mathrm{ns}}$ & $6,32^{\mathrm{ns}}$ & $1,05^{\mathrm{ns}}$ & $4,84^{\mathrm{ns}}$ & $0,68^{\mathrm{ns}}$ & $1,90^{\mathrm{ns}}$ & $0,40^{\mathrm{ns}}$ \\
\hline DMS & 1,07 & 0,41 & 0,07 & 0,34 & 0,11 & 1,09 & 0,09 \\
\hline CV $(\%)$ & 7,57 & 7,57 & 7,73 & 7,45 & 16,97 & 14,54 & 3,38 \\
\hline
\end{tabular}

Médias provenientes de quatro repetições composta de quatro buquês de cinco frutos. Médias seguidas pela mesma letra na coluna não diferem entre si, ao nível de 5\% de probabilidade pelo teste F. Unidades: Perda de Peso (\%), Cor (índice de 1 a 7), Ácido Málico (\%), Clorofilas $\left(\mathrm{mg}_{\mathrm{g}} \mathrm{cm}^{-2} 10^{-4}\right) \mathrm{e}$ Carotenóides $\left(\mathrm{mg} . \mathrm{cm}^{-2} 10^{-4}\right)$.

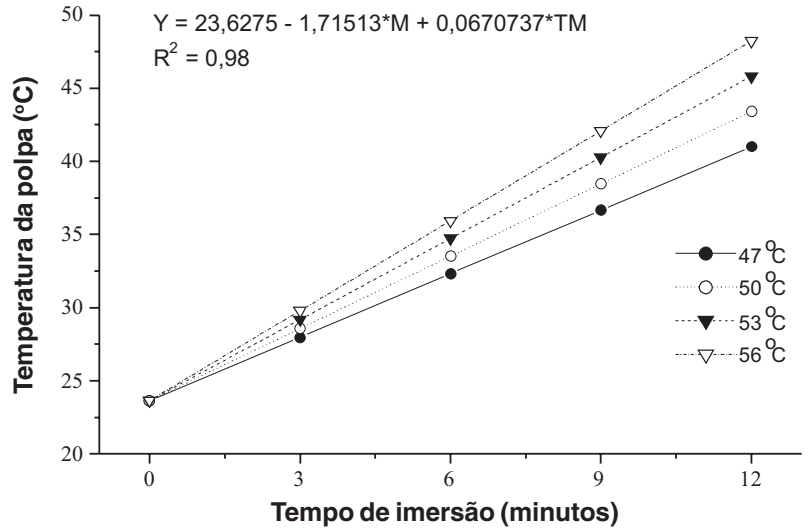

FIG. 1 - Estimativa da temperatura central da polpa $(Y)$ de frutos de banana (Musa spp.) 'Prata anã' submetidos à termoterapia, em função da temperatura da água $(T)$ e do tempo de imersão $(M)$.

melhores tratamentos foram $56^{\circ} \mathrm{C}$ durante três a 9 min, nos quais, a \% ALF manteve-se nula (Tabela 4).

Em geral, a\% ALF tende ser reduzida consideravelmente com o aumento da temperatura da água e do tempo de imersão (Tabela 4). As médias apresentadas indicaram que frutos inoculados com C. musae e tratados a $56{ }^{\circ} \mathrm{C}$, durante 3, 6 e 9 min, não apresentaram podridões aos 12 dias após os tratamentos, enquanto que os não tratados (0 min) apresentaram em média 64\% de ALF. Decorrido o período de avaliação, apenas os frutos tratados a $56^{\circ} \mathrm{C}$, durante 6 e 9 min permaneceram livres de podridões, enquanto aqueles tratados a $56{ }^{\circ} \mathrm{C}$ durante 3 e $12 \mathrm{~min}$ apresentaram algum nível de lesão. Se por um lado o tratamento a $56{ }^{\circ} \mathrm{C}$ durante 9 min evitou a manifestação de podridões, por outro promoveu injúrias na casca dos frutos.

$\mathrm{O}$ tratamento $56^{\circ} \mathrm{C}$ durante 12 min não foi efetivo no controle de podridões, aos 12 dias após o tratamento pois,
TABELA 2 - Percentagem de germinação de esporos de Colletotrichum musae submetidos à termoterapia e incubados sob condições ambientes, em função da temperatura da água e do tempo de imersão, 48 h após o tratamento

\begin{tabular}{cccccc}
\hline \hline \multirow{2}{*}{$\begin{array}{c}\text { Tempo } \\
\text { (minutos) }\end{array}$} & $\mathbf{4 7}$ & $\mathbf{5 0}$ & $\mathbf{5 3}$ & $\mathbf{5 6}$ & Médias \\
\cline { 2 - 5 } & $90,30 \mathrm{Aa}$ & $90,30 \mathrm{Aa}$ & $90,30 \mathrm{Aa}$ & $90,30 \mathrm{Aa}$ & $90,30 \mathrm{~A}$ \\
0 & $98,47 \mathrm{Aa}$ & $98,32 \mathrm{Aa}$ & $19,41 \mathrm{Bb}$ & $0,00 \mathrm{Bc}$ & $54,05 \mathrm{~B}$ \\
3 & $96,41 \mathrm{Aa}$ & $88,69 \mathrm{Aa}$ & $3,72 \mathrm{Bb}$ & $0,00 \mathrm{Bb}$ & $47,20 \mathrm{~B}$ \\
6 & $98,04 \mathrm{Aa}$ & $96,89 \mathrm{Aa}$ & $0,00 \mathrm{Bb}$ & $0,00 \mathrm{Bb}$ & $48,73 \mathrm{~B}$ \\
9 & $97,38 \mathrm{Aa}$ & $92,82 \mathrm{Aa}$ & $0,00 \mathrm{Bb}$ & $0,00 \mathrm{Bb}$ & $47,55 \mathrm{~B}$ \\
12 & $96,12 \mathrm{a}$ & $93,40 \mathrm{a}$ & $22,68 \mathrm{~b}$ & $18,06 \mathrm{~b}$ & \\
\hline Médias & &
\end{tabular}

Médias originais provenientes de três repetições por tratamento. As médias seguidas pela mesma letra maiúscula na coluna e médias seguidas pela mesma letra minúscula na linha, não diferem entre si ao nível de 5\% de probabilidade pelo teste de Tukey. CV $(\%)=18,81$.

além de promover injúrias nas casca, alterou a suscetibilidade dos frutos a outros patógenos oportunistas que não foram afetados pela termoterapia ou favoreceu a recontaminação por C. musae (Tabela 4). Portanto, este fato representa a maior limitação ao uso da termoterapia, ou seja, a falta de proteção residual contra a recontaminação por patógenos oportunistas e injúrias promovidas no hospedeiro (Edney \& Burchill, 1967). Resultados positivos com termoterapia foram obtidos à temperatura de $50{ }^{\circ} \mathrm{C}$ durante $20 \mathrm{~min}$, com redução da podridão da coroa, causada por $C$. paradoxa em banana, de $100 \%$ para menos de $3 \%$ (Reyes et al., 1998). Infecções latentes induzidas artificialmente por $C$. musae em bananas foram também controladas pela imersão de frutos verdes em água a $55^{\circ} \mathrm{C}$ por 2 min e 50 a $54{ }^{\circ} \mathrm{C}$ por 5 min (Burden, 1968).

O tratamento $56{ }^{\circ} \mathrm{C}$, durante $6 \mathrm{~min}$, não paralisou o crescimento micelial in vitro (Tabela 3 ), porém mostrou-se 
Termoterapia de banana 'Prata-Anã' no controle de podridões...

TABELA 3 - Crescimento micelial (cm) de Colletotrichum musae submetido à termoterapia e incubado sob condições ambientes, em função da temperatura da água e do tempo de imersão seis dias após o tratamento

\begin{tabular}{|c|c|c|c|c|c|}
\hline \multirow{2}{*}{$\begin{array}{c}\text { Tempo } \\
\text { (minutos) }\end{array}$} & \multicolumn{4}{|c|}{ Temperatura $\left({ }^{\circ} \mathrm{C}\right)$} & \multirow{2}{*}{ Médias } \\
\hline & 47 & 50 & 53 & 56 & \\
\hline 0 & $8,50 \mathrm{Aa}$ & $8,50 \mathrm{Aa}$ & $8,50 \mathrm{Aa}$ & $8,50 \mathrm{Aa}$ & $8,50 \mathrm{~A}$ \\
\hline 3 & $8,50 \mathrm{Aa}$ & $8,50 \mathrm{Aa}$ & $8,50 \mathrm{Aa}$ & $8,50 \mathrm{Aa}$ & $8,50 \mathrm{~A}$ \\
\hline 6 & $8,50 \mathrm{Aa}$ & $8,50 \mathrm{Aa}$ & $8,50 \mathrm{Aa}$ & $8,50 \mathrm{Aa}$ & $8,50 \mathrm{~A}$ \\
\hline 9 & $8,50 \mathrm{Aa}$ & $8,50 \mathrm{Aa}$ & $8,50 \mathrm{Aa}$ & $8,50 \mathrm{Aa}$ & $8,50 \mathrm{~A}$ \\
\hline 12 & $8,50 \mathrm{Aa}$ & $8,50 \mathrm{Aa}$ & $8,50 \mathrm{Aa}$ & $4,81 \mathrm{Bb}$ & $7,57 \mathrm{~B}$ \\
\hline Médias & $8,50 \mathrm{a}$ & $8,50 \mathrm{a}$ & $8,50 \mathrm{a}$ & $7,76 \mathrm{~b}$ & \\
\hline
\end{tabular}

Médias originais provenientes de três repetições por tratamento. As médias seguidas da mesma letra maiúscula na coluna e médias seguidas pela mesma letra minúscula na linha, não diferem entre si ao nível de 5\% de probabilidade pelo teste de Tukey. CV $(\%)=8,20$.

efetivo no controle das podridões in vivo (Tabela 4). A eficiência deste tratamento pode, provavelmente, ter sido favorecida pelas condições ambientes, com temperatura variando de 15 a $23{ }^{\circ} \mathrm{C}$ e umidade relativa de 60 a $85 \%$, adversas ao desenvolvimento dos patógenos e predominantes no período e local do experimento. Neste período, portanto, esse tratamento se mostrou altamente eficiente no controle das podridões em banana, sem alterar a qualidade dos frutos e com potencial para substituir os fungicidas.

No experimento instalado no verão, quando a temperatura ambiente variou de 23 a $30^{\circ} \mathrm{C}$, pôde-se observar outro problema da termoterapia, uma vez que o tratamento a $56{ }^{\circ} \mathrm{C}$ durante $6 \mathrm{~min}$, além de provocar indícios de escurecimento da casca nas extremidades dos frutos, não apresentou controle total das podridões, resultando em $11 \%$ de ALF (dados não apresentados). Esse resultado se contrapõe ao obtido no mês de maio, onde os frutos tratados a $56{ }^{\circ} \mathrm{C}$ durante 6 min não foram afetados por podridões e nem exibiram injúrias na casca. Desta forma, pode-se sugerir que a eficiência da termoterapia no controle da podridão e a sensibilidade dos frutos foi influenciada pelas condições ambientes, durante a estação de crescimento dos frutos e da colheita dos cachos.

Diferenças na eficiência da termoterapia também foram observadas por Shiffmann-Nadel \& Cohen (1966) em frutos produzidos em diferentes temperaturas de crescimento e práticas culturais, de diferentes cultivares e colhidos em diferentes estádios de maturidade. As respostas diferenciais pelo hospedeiro também foram caracterizadas por injúrias na casca dos frutos. Relatos de Paull (1994) indicaram que a temperatura média mínima superior a $22,4{ }^{\circ} \mathrm{C}$, nos últimos três dias antes da colheita dos cachos, esteve mais relacionada à presença de injúrias em frutos de mamão (Carica papaya L.) tratados com água quente. Golan \& Phillips (1991), demonstraram que o fruto pode ser protegido das injúrias com o pré-condicionamento, a exemplo de limões acondicionados por dois a oito dias a $15,5^{\circ} \mathrm{C}$, que se mostraram mais tolerantes à termoterapia.
TABELA 4 - Percentagem da área lesionada / fruto (\% ALF) em frutos de banana 'Prata anã' (Musa spp.) submetidos à termoterapia e incubados sob condições ambientes, em função da temperatura da água e do tempo de imersão, 12 dias após o tratamento

\begin{tabular}{cccccc}
\hline \hline Tempo & \multicolumn{4}{c}{ Temperatura (C) } & \multirow{2}{*}{ Me dias } \\
\cline { 2 - 5 } (minutos) & $\mathbf{4 7}$ & $\mathbf{5 0}$ & $\mathbf{5 3}$ & $\mathbf{5 6}$ & \\
\hline 0 & $63,66 \mathrm{BCa}$ & $63,33 \mathrm{Aa}$ & $64,00 \mathrm{Aa}$ & $64,00 \mathrm{Aa}$ & $63,75 \mathrm{~A}$ \\
3 & $89,33 \mathrm{Aa}$ & $49,67 \mathrm{Bc}$ & $64,00 \mathrm{Ab}$ & $0,00 \mathrm{Cd}$ & $50,75 \mathrm{~B}$ \\
6 & $64,00 \mathrm{Ba}$ & $64,00 \mathrm{Aa}$ & $64,00 \mathrm{Aa}$ & $0,00 \mathrm{Cb}$ & $48,00 \mathrm{C}$ \\
9 & $64,33 \mathrm{Ba}$ & $64,00 \mathrm{Aa}$ & $12,00 \mathrm{Bb}$ & $0,00 \mathrm{Cc}$ & $35,08 \mathrm{E}$ \\
12 & $60,00 \mathrm{Cb}$ & $45,00 \mathrm{Cc}$ & $64,00 \mathrm{Aa}$ & $8,00 \mathrm{Bd}$ & $44,25 \mathrm{D}$ \\
\hline Medias & $68,26 \mathrm{a}$ & $57,20 \mathrm{~b}$ & $53,60 \mathrm{c}$ & $14,40 \mathrm{~d}$ & \\
\hline
\end{tabular}

Médias transformadas por raiz $(x+1)$ provenientes de 16 buquês de cinco frutos. As médias seguidas da mesma letra maiúscula na coluna e médias seguidas da mesma letra minúscula na linha, não diferem entre si ao nível de $5 \%$ de probabilidade pelo teste de Tukey. CV $(\%)=3,58$.

Os dados meteorológicos registrados durante a estação de crescimento dos frutos indicaram médias de temperatura mínima, nos três, sete, dez e 18 dias, anteriores à colheita dos cachos, de $15{ }^{\circ} \mathrm{C}$ em maio e de $18{ }^{\circ} \mathrm{C}$ em novembro. A temperatura dos frutos, durante a termoterapia realizada em novembro, mostrou-se, portanto, superior à dos frutos tratados em maio, sendo as injúrias na casca observadas a $56^{\circ} \mathrm{C}$ por 9 min em maio, e 6 min em novembro. Em novembro, a temperatura dos frutos mostrou-se mais próxima à temperatura de equilíbrio, necessitando de menor tempo de exposição para promover injúrias.

Sendo assim, a termoterapia a $56{ }^{\circ} \mathrm{C}$ por $6 \mathrm{~min}$, mostrou-se suficiente para controlar as podridões em frutos de banana, sem causar injúrias em locais e períodos com predominância de baixas temperaturas. Entretanto, para condições de elevadas temperaturas, a termoterapia apenas reduziu a\% de ALF para $11 \%$.

Os resultados obtidos mostraram que os efeitos benéficos de combinações de tempo de tratamento e temperatura da água não podem ser generalizados, uma vez que fatores de pré-colheita influenciam na eficiência do tratamento. Desse modo, a termoterapia pode ser recomendada para controle de podridão em pós-colheita de banana devendo, porém, ser ajustada para diferentes estações do ano.

\section{REFERÊNCIAS BIBLIOGRÁFICAS}

ARMSTRONG, J.W. Development of a hot water immersion quarantine treatment for Hawaiian grown 'Brazilian' bananas. Journal of Economic Entomology 75:787-791. 1982.

BARKAI-GOLAN, R. \& PHILLIPS, D.J. Postharvest heat treatment of fresh fruit and vegetables for decay control. Plant Disease 75:1085-1089. 1991.

BLEINROTH, E.W. (Ed.). Tecnologia de pós-colheita de frutos tropicais. Campinas: ITAL, Manual Técnico 9. 1992. 203p.

BURDEN, O.J. Reduction of banana anthracnose following hot- 
water treatment of the green fruit. Queesland Journal of Agric. And Animal Sciences 25:135-144. 1968.

COATES, L. \& GOWANLOCK, D. Infection process of Colletotrichum species in subtropical and tropical fruits. Proceedings, Postharvest handling of tropical fruits. Australia. 1994, pp.162-168.

COUEY, M.H. Heat treatment for control postharvest disease and insect pest of fruits. HortScience 24:198-202. 1989.

DADZIE, B.K. \& ORCHARD, J.E. Postharvest criteria and methods for routine screening of banana/plantain hybrids. Proceedings, International Network for Improvement of Banana and Plantain, Montpellier, France, 1996.

EDNEY, K.L. \& BURCHILL, R.T. The use of heat to control the rotting of Cox's orange apples by Gloeosporium. Annual Applied Biology 59:389-400. 1967.

FAO. FAOSTAT. Disponível em:<http://www.fao.org/ag/guides/ resource/data.htm>. Acesso em:21. nov. 2004.

GOLAN, R. B. \& PHILLIPS, D.J. Postharvest heat treatment of fresh fruits and vegetables for decay control. Plant Disease 75:1085-1089. 1991.

JACOBI, K.K. \& GILES, J.E. Quality of 'Kensington'mango (Mangifera indica Linn.) fruit following combined vapour heat desinfestation and water disease control treatments. Postharvest Biology and Technology 12:285-292. 1997.

JESUS, W.C., BENATO, E.A. \& SOUZA, N.L. Tratamento térmico pós-colheita de frutos de maracujazeiro para controle de antracnose (Colletotrichum gloeosporioides). Fitopatologia Brasileira 19:283. 1994 (Resumo).
JOHNSON, G.I. \& SANGHOTE, S. Control postharvest disease of tropical fruits: challenges for the 21st century. Proceedings, Postharvest handling of tropical fruits. Australia. 1994. pp.140161.

LIU, X., GUO. G., HUANG, S., LIU, X.J., HUANG, S.M. \& GUO, G. The research and utilization of postharvest heat treatment for fruit storage. South China Fruits 26:46-48. 1997.

PLOETZ, R., ZENTMYER, G.A., NISHIJIMA, W.T., ROHRBACH, K.G. \& OHR, H.D. (Eds). Compendium of tropical fruit diseases, Minnesota-USA, APS Press, 1994.

PAULL, R.E. Tropical fruit physiology and storage potencial. In: Champp, B.R., Higley, E. \& Jonson, G.I. (Eds.) Postharvest handling of tropical fruits. Australia, 1994. Proceedings... Australia: ACIAR 50:198-204. 1994.

RAMMA, I., MADHU, S.P.B. \& PEERTHUM, P. Postharvest quality improvement of banana. Food and Agricultural Research Council: 187-194. 1999.

RAHMAN, R.A., GRANDISON, A., \& CAMPBALL PLATT, G. Electron beam irradiation combined with hot-water immersion treatment for banana preservation. Proceedings, Postharvest handling of tropical fruits, Australia. 1994. p.378.

REYES, M.E.Q., NISHIJIMA, W. \& PAULL, R. Control of crowm rot in 'Santa Catarina Prata' and 'Williams' banana with hot water treatments. Postharvest Biology and Technology 14:71-75. 1998. SHIFFMANN-NADEL, M. \& COHEN, E. Influence of growth temperatures on the effectiveness of heat treatment of Phytophthora- infected citrus fruits. Plant Disease Reporter 50:867-868. 1966. 